\title{
Construction of $\theta$-Cyclic Codes over an Algebra of Order 4
}

\author{
Irwansyah $^{1 *}$, Intan Muchtadi-Alamsyah ${ }^{1}$, Aleams Barra ${ }^{1}$, Ahmad Muchlis $^{1}$, Djoko Supriyanto ${ }^{2}$ \\ ${ }^{\text {I}}$ Algebra Research Group, Bandung Institute of Technology, Indonesia. \\ ${ }^{2}$ Combinatorial mathematic research group, Institut Teknologi Bandung, Indonesia.
}

Received: 20 September 2014 / Accepted: 30 November 2014

\begin{abstract}
:
In this paper, we show that $\theta$-cyclic codes over algebra $A_{1}=\mathrm{F}_{2}+v \mathrm{~F}_{2}$ can be constructed from binary cyclic codes using a bijection map from $A_{1}$ to $\mathrm{F}_{2} \times \mathrm{F}_{2}$. We also give a decoding algorithm for $\theta$-cyclic codes which are constructed using well-known $\mathrm{BCH}$ codes over binary field.
\end{abstract}

Key words: $B C H$ codes, Berlekamp-Massey decoding algorithm, binary cyclic codes, $\theta$-cyclic codes, gray map

\section{Introduction}

Cyclic codes has many applications in data storage systems e.g. QR code, DVD, satellite communications etc. Therefore, many researchers put an attempt to study this code and its generalizations in order to find this type of codes with better parameter. $\theta$-cyclic codes or skew cyclic codes as a generalization of cyclic codes, has been extensively studied by researcher because it produces some codes with better parameter and has a nice structure.

Boucher and Ulmer [3] studied the structure of skew cyclic codes over Galois field $\mathrm{F}_{q}$ and using Gröbner bases, they compute all Euclidean and Hermitian selfdual $\theta$-cyclic codes over $\mathrm{F}_{4}$ of length less than 40, including a $[36,18,11]$ Euclidean self-dual $\theta$-cyclic code which improve the previously best known bound. In [1], Abualrub et al. studied $\theta$-cyclic codes over algebra $A_{1}=\mathrm{F}_{2}+v \mathrm{~F}_{2}$, where $v^{2}=v$. They gave the generators for every $\theta$-cyclic codes over $A_{1}$ and characterized the generator of Euclidean and Hermitian $\theta$-cyclic codes over $A_{1}$. Furthermore, Gao [5] studied the structure of $\theta$-cyclic codes over $\mathrm{F}_{p}+v \mathrm{~F}_{p}$, where $v^{2}=v$ and $p$ is a prime number for special type of $\theta$. $\mathrm{He}$ found that $\theta$-cyclic codes over $\mathrm{F}_{p}+v \mathrm{~F}_{p}$ are equivalent to either cyclic codes or quasi-cyclic codes and gave the enumeration of distinct $\theta$-cyclic codes over $\mathrm{F}_{p}+v \mathrm{~F}_{p}$.
In this paper, we study $\theta$-cyclic codes over $A_{1}$ with different point of view with Abualrub et al. [1]. Our method gives a way to construct this codes from binary cyclic codes, so we can easily determine the size and minimum distance of $\theta$-cyclic codes over $A_{1}$.

\section{Construction of $\theta$-cyclic codes over $A_{1}$}

Let $A_{1}=\mathrm{F}_{2}+v \mathrm{~F}_{2}=\{0,1, v, v+1\}$, where $v^{2}=v$. Indeed, it is an algebra over binary field $\mathrm{F}_{2}$ of order 4 . $A_{1}$ has a unique non-trivial automorphism $\theta$, where $\theta(\alpha+\beta v)=\alpha+\beta+\beta v$ for $\alpha, \beta \in \mathrm{F}_{2} . C$ is a $\theta$ cyclic codes of length $n$ over $A_{1}$ if it is a submodule of $A_{1}^{n}$ over $A_{1}$ and if $c=\left(c_{0}, c_{1}, \ldots, c_{n-1}\right) \in C$, then $T_{\theta}(c)=\left(\theta\left(c_{n-1}\right), \theta\left(c_{0}\right), \ldots, \theta\left(c_{n-2}\right)\right) \in C$ also. Let $\varphi$ is a Gray map from $A_{1}$ to $\mathrm{F}_{2} \times \mathrm{F}_{2}$, where $\varphi(\alpha+\beta v)=(\alpha, \alpha+\beta)$ for $\alpha, \beta \in \mathrm{F}_{2}$. Using the inverse of Gray map $\varphi$, we have the following results.

Proposition 1. If $C_{1}$ is a binary cyclic code of length $n$, then $\varphi^{-1}\left(C_{1}, C_{1}\right)$ is a $\theta$-cyclic code over $A_{1}$ of length $n$.

Proof. For any $c_{1}, c_{2} \in C_{1}$, consider $\varphi^{-1}\left(c_{1}, c_{2}\right)$. We will show that there exist $c_{3}, c_{4} \in C_{1}$ such that 
$T_{\theta}\left(\varphi^{-1}\left(c_{1}, c_{2}\right)\right)=\varphi^{-1}\left(c_{3}, c_{4}\right)$

Let

$c_{1}=\left(c_{10} c_{11} \ldots c_{1 n-1}\right)$ and $c_{1}=\left(c_{20} c_{21} \ldots c_{2 n-1}\right)$.

Then, we have

$\varphi^{-1}\left(c_{1}, c_{2}\right)=\left(c_{10}+\left(c_{10}+c_{20}\right) v \ldots c_{10}+\left(c_{1 n-1}+c_{2 n-1}\right) v\right)$

So,

$\varphi\left(c=T_{\theta}\left(\varphi^{-1}\left(c_{1}, c_{2}\right)\right)\right)=\left(\begin{array}{llll}c_{2 n-1} & c_{20} & \cdots & c_{2 n-2} \\ c_{1 n-1} & c_{10} & \cdots & c_{1 n-2}\end{array}\right)$

Then, let $c_{i}^{1}=\left(c_{i n-1} c_{i 0} \ldots c_{i n-2}\right)$, for $i=1,2$. Choose $c_{3}=c_{2}^{1} \quad$ and $\quad c_{4}=c_{1}^{1} \quad$, we have $\varphi^{-1}\left(c_{3}, c_{4}\right)=c=T_{\theta}\left(\varphi^{-1}\left(c_{1}, c_{2}\right)\right)$. Hence, $\varphi^{-1}\left(C_{1}, C_{1}\right)$ is a $\theta$-cyclic code. $Q E D$

Let $T(C)$ is the set of first shift of all code words in $C$, i.e. if $c=\left(c_{0}, \ldots, c_{n-1}\right) \in C \quad$, then $T(c)=\left(c_{n-1}, c_{0}, \ldots, c_{n-2}\right)$. We have the following weak characterization.

Proposition 2. $C$ is a $\theta$-cyclic code over $A_{1}$, if and only if $C=\varphi^{-1}\left(C_{0}, C_{1}\right)$, where $C_{0}$ and $C_{1}$ are binary quasicyclic codes of index 2 such that $T\left(C_{i}\right)=C_{i+1} \bmod 2$ for $i=0,1$.

Proof. ( $\Leftarrow)$ ) For any $c \in C$, there exist codewords $c_{1} \in C_{0}$ and $c_{2} \in C_{1}$ such that $c=\varphi^{-1}\left(c_{1}, c_{2}\right)$. Let $c_{i}=\left(c_{i 0}, \ldots, c_{i n-1}\right)$ for $i=0,1$. Then, we have

$$
c=\left(c_{00}+\left(c_{00}+c_{10}\right) v, \ldots, c_{0 n-1}+\left(c_{0 n-1}+c_{1 n-1}\right) v\right)
$$

we will show that $T_{\theta}(c) \in C$. We can see that,

$T_{\theta}(c)=\left(c_{0 n-1}+\left(c_{0 n-1}+c_{1 n-1}\right) v, \ldots, c_{0 n-2}+\left(c_{0 n-2}+c_{1 n-2}\right) v\right)$

So, if we choose $c_{3}=T\left(c_{2}\right) \in C_{0}$ and $c_{4}=T\left(c_{1}\right) \in C_{1}$, we have $\varphi^{-1}\left(c_{3}, c_{4}\right)=T_{\theta}(c)$. Therefore, $C$ is a cyclic code. The other direction also follows similarly. $Q E D$

Theorem 1. For $n$ is an odd integer, $C$ is a $\theta$-cyclic code over $A_{1}$ of length $n$ if and only if $C=\varphi^{-1}\left(C_{1}, C_{1}\right)$, where $C_{1}$ is a binary cyclic code.

Proof. Let $C=\varphi^{-1}\left(C_{1}, C_{2}\right)$. For any $c_{1} \in C_{1}$, let $c_{1}=\left(c_{10} \ldots c_{1 n-1}\right)$, consider

$\varphi^{-1}\left(c_{1}, 0\right)=\left(c_{10}+c_{10} v \ldots c_{1 n-1}+c_{1 n-1} v\right)$ we can see that,

$T_{\theta}^{i}\left(\varphi^{-1}\left(c_{1}, 0\right)\right)= \begin{cases}\left(c_{1,0+i}+c_{1,0+i} v, \ldots, c_{1, n-1+i}+c_{1, n-1+i} v\right), & \text { i even } \\ \left(c_{1,0+i} v_{1} \ldots, c_{1, n-1+i} v\right), & \text { iodd }\end{cases}$

so

$T_{\theta}^{n+1}\left(\varphi^{-1}\left(c_{1}, 0\right)\right)=\left(c_{1 n-1}+c_{1 n-1} v, c_{10}+c_{10} v \ldots c_{1 n-2}+c_{1 n-2} v\right)$

Then, if we choose $c_{3}=c_{1}^{1}$, then we have $\varphi^{-1}\left(c_{3}, 0\right)=T_{\theta}^{n+1}\left(\varphi^{-1}\left(c_{1}, 0\right)\right) \in C$, and $c_{3}=c_{1}^{1} \in C_{1}$, which means $C_{1}$ is a binary cyclic code. Conclusion for $C_{2}$ we obtain in similar ways.

For any $c_{1} \in C_{1}$, let $c_{1}=\left(c_{10} \ldots c_{1 n-1}\right)$. By equation for $T_{\theta}^{i}\left(\varphi^{-1}\left(c_{1}, 0\right)\right)$, we have

$$
\varphi\left(T_{\theta}^{n}\left(c_{1}, 0\right)\right)=\left(\begin{array}{cccc}
0 & 0 & \cdots & 0 \\
c_{10} & c_{11} & \cdots & c_{1 n-1}
\end{array}\right)=\varphi^{-1}\left(0, c_{1}\right)
$$

Consequently, $c_{1} \in C_{2}$, which implies $C_{1} \subseteq C_{2}$. By similar arguments, we also have $C_{2} \subseteq C_{1}$. Therefore, $C_{1}=C_{2} \cdot Q E D$

Proposition 3. If $C=\varphi^{-1}\left(C_{1}, C_{2}\right)$ is a $\theta$-cyclic codes over $A_{1}$, where $C_{2} \subseteq C_{1}$ and $n$ is an even integer, then $C_{1}$ is a binary cyclic code.

Proof. Any $c_{1} \in C_{1}$, we can let $c_{1}=\left(c_{10} \ldots c_{1 n-1}\right)$, then we have

$T_{\theta}\left(\varphi^{-1}\left(c_{1}, 0\right)\right)=\left(\begin{array}{ccc}0 & \cdots & 0 \\ c_{1 n-1} & \cdots & c_{1 n-2}\end{array}\right)$

which implies $c_{1}^{1} \in C_{1} \cdot Q E D$

Proposition 4. If $C=\varphi^{-1}\left(C_{1}, C_{2}\right)$ is a $\theta$-cyclic codes over $A_{1}$, where $C_{1}$ and $C_{2}$ are binary cyclic codes, then $C_{2}=C_{1}$.

Proof. Take any $c_{1} \in C_{1}$, and let $c_{1}=\left(c_{10} \ldots c_{1 n-1}\right)$, we can see that

$\varphi\left(T_{\theta}\left(\varphi^{-1}\left(c_{1}^{n-1}, 0\right)\right)\right)=\left(\begin{array}{cccc}0 & 0 & \cdots & 0 \\ c_{10} & c_{11} & \cdots & c_{1 n-1}\end{array}\right)$

so, $C_{1} \subseteq C_{2}$. By similar arguments, we also have $C_{2} \subseteq C_{1}$. Hence, $C_{1}=C_{2}$. QED 
We also have several direct consquences for Euclidean and Hermitian $\theta$-cyclic codes over $A_{1}$ which can be proved using our results combined with Lemma 3.1 and Lemma 3.2 in [2].

Corollary 1. If $C_{1}$ is a binary self-dual cyclic code, then $C=\varphi^{-1}\left(C_{1}, C_{1}\right)$ is an Euclidean and Hermitian $\theta$ cyclic code over $A_{1}$.

Corollary 2. If $C=\varphi^{-1}\left(C_{1}, C_{2}\right)$ is an Euclidean $\theta$ cyclic codes over $A_{1}$, where $C_{2} \subseteq C_{1}$ and $n$ is an even integer, then $C_{1}$ is a binary self-dual cyclic code.

Corollary 3. If $C=\varphi^{-1}\left(C_{1}, C_{2}\right)$ is a Hermitian $\theta$ cyclic codes over $A_{1}$, where $C_{2} \subseteq C_{1}$ and $n$ is an even integer, then $C_{1}$ is a binary self-dual cyclic code and $C_{1}=C_{2}$.

The above results show that we can construct a $\theta$-cyclic code $C$ over $A_{1}$ using a binary cyclic code $C_{1}$ simply using relation $C=\varphi^{-1}\left(C_{1}, C_{1}\right)$, and we can easily know that minimum distance of $C$ is equals to minimum distance of $C_{1}$ and size of $C$ is equals to square of the size of $C_{1}$.

\section{Decoding algorithm}

If $C_{1}$ is a binary $\mathrm{BCH}$ code of length $n=2^{m}-1$ for some $m$, then we can have decoding algorithm for $\theta$ cyclic code $C=\varphi^{-1}\left(C_{1}, C_{1}\right)$ simply using decoding algorithm of $\mathrm{BCH}$ codes with some additional steps. Note that, $n=2^{m}-1$ is an odd integer, thats why we only need one binary $\mathrm{BCH}$ code to construct $\theta$-cyclic over $A_{1}$ in this situation. The simplified algorithm is as follows.
1. Let $c^{\prime}$ be the original message, but vector $c \in A_{1}^{n}$ received. Then calculate $\varphi(c)=\left(c_{1}, c_{2}\right)$ to have $c_{1}, c_{2} \in \mathrm{F}_{2}^{n}$.

2. Apply decoding algorithm for $\mathrm{BCH}$ codes to each $c_{1}$ and $c_{2}$ to get $c_{1}{ }^{\prime}, c_{2}{ }^{\prime} \in C_{1}$.

3. Calculate $\varphi^{-1}\left(c_{1^{\prime}}, c_{2^{\prime}}\right)$, and $c^{\prime}=\varphi^{-1}\left(c_{1}{ }^{\prime}, c_{2}{ }^{\prime}\right)$ is the original message.

\section{Conclusions}

We can construct several $\theta$-cyclic codes over $A_{1}$ using binary cyclic codes, so as a consequence, we can easily determine its minimum distance and size. We are able to apply decoding algorithm for binary $\mathrm{BCH}$ codes to decode $\theta$-cyclic codes which constructed using binary $\mathrm{BCH}$ code also.

\section{Acknowledgement}

This research is supported by Beasiswa Unggulan BPKLN DIKTI through Program Doktor Unggulan Batch 3-B FMIPA ITB and Hibah Riset dan Inovasi KK ITB.

\section{References}

[1] T. Abualrub, N. Aydin, and P. Seneviratne, On $\theta$-Cyclic Codes over $\mathrm{F}_{2}+v \mathrm{~F}_{2}$, Australasian Journal of Combinatorics, 54, 2012, 115-126.

[2] K. Betsumiya and M. Harada, Optimal self-dual codes over $\mathrm{F}_{2} \times \mathrm{F}_{2}$ with respect to the Hamming weight, IEEE Transactions on Information Theory, 50(2), 2004, 356-358.

[3] D. Boucher and F. Ulmer, Coding with Skew Polynomial Rings, Journal of Symbolic Computation, 44, 2009, 16441656.

[4] D. Boucher, W. Geiselmann, and F. Ulmer, Skew Cyclic Codes, Appl. Algebra Eng. Commun. Comput., 18, 2007, 379-389.

[5] J. Gao, Skew Cyclic Codes over $\mathrm{F}_{p}+v \mathrm{~F}_{p}$, J. Appl. Math. and Informatics, 31(3-4), 2013, 337-342. 